(2) Open Access Full Text Article

\title{
Hepatic artery-infusion chemotherapy improved survival of hepatocellular carcinoma after radical hepatectomy
}

\author{
Min Feng' \\ Chengwu Tang' \\ Wenming Feng' \\ Ying Bao' \\ Yinyuan Zheng ${ }^{2}$ \\ Jianbin Shen' \\ 'Department of General Surgery, \\ ${ }^{2}$ Department of Radiology, \\ First People's Hospital, Huzhou \\ University Medical College, Huzhou, \\ Zhejiang, China
}

This article was published in the following Dove Press journal:

OncoTargets and Therapy

14 June 2017

Number of times this article has been viewed

Objective: To evaluate the effect of postoperative hepatic artery-infusion chemotherapy (HAIC) on survival probability in patients with hepatocellular carcinoma (HCC) after radical hepatectomy.

Patients and methods: This retrospective study included 85 patients with HCC who received radical hepatectomy from May 2005 to May 2010. Among these patients, 42 underwent two sessions of HAIC (5-fluoruracil [1,000 $\left.\mathrm{mg} / \mathrm{m}^{2}\right]$, oxaliplatin $\left[85 \mathrm{mg} / \mathrm{m}^{2}\right]$, and mitomycin-C $\left[6 \mathrm{mg} / \mathrm{m}^{2}\right]$ ) after radical hepatectomy (HAIC group), and 43 underwent radical hepatectomy only (the control group). HAIC-related side effects and long-term survival were retrospectively analyzed.

Results: The HAIC group showed a significantly higher 5-year intrahepatic recurrence-free survival probability and lower risk of intrahepatic recurrence (HR $0.5615,95 \%$ CI $0.3234-0.9749$ [log-rank test]; $P=0.0332$ ). The HAIC group also had significantly higher 5-year disease-free survival probability (HR 0.591, 95\% CI 0.3613-0.9666 [log-rank test]; $P=0.0298$ ) and overall survival probability than the control group (HR 0.5768, 95\% CI 0.3469-0.9589 [log-rank test]; $P=0.0278$ ). No HAIC-related deaths in the HAIC group were reported. All toxicities and complications were controlled, and no patients quit the treatment.

Conclusion: HAIC can effectively and safely reduce intrahepatic recurrence and improve the long-term survival of patients with HCC after radical hepatectomy.

Keywords: hepatocellular carcinoma, hepatic artery-infusion chemotherapy, intrahepatic recurrence, metastasis

\section{Introduction}

Hepatocellular carcinoma (HCC) leads to 690,000 deaths annually worldwide. More than half of these deaths occur in China, and thus the disease is considered the secondmost fatal cancer in the country. ${ }^{1}$ Various treatment options are currently available, such as surgical resection, radiofrequency ablation, percutaneous ethanol injection, transcatheter arterial chemoembolization, radiotherapy, and hepatic artery-infusion chemotherapy (HAIC). ${ }^{2}$

Radical resection is the optimal therapy for patients with $\mathrm{HCC}$ with early disease stage and good liver-function reserve. ${ }^{3,4}$ However, more than $80 \%$ of patients with $\mathrm{HCC}$ develop recurrence within 5 years after radical hepatectomy, which significantly harms long-term survival. ${ }^{5-7}$ Postoperative recurrence of $\mathrm{HCC}$ is related to multicenter growth of tumor and microscopic tumor thrombosis. ${ }^{8,9}$ Both univariate and multivariate analyses of the prognostic factors indicate that intrahepatic recurrence significantly affects survival. ${ }^{10,11}$ Macroscopic tumor foci are resected by surgery; however,
Correspondence: Jianbin Shen Department of General Surgery, First People's Hospital, Huzhou University Medical College, I58 Guangchang Hou Road, Huzhou, Zhejiang 313000, China Tel +86572 2039347

Fax +865722023728

Email shenjianbin_dr@aliyun.com 
undetected residual microscopic foci will then lead to relapse. ${ }^{8}$ Therefore, elimination of the residual microscopic foci plays an important role in improving the long-term survival of HCC after surgery. The present study aims to determine the safety and efficacy of HAIC in patients with $\mathrm{HCC}$ after radical hepatectomy.

\section{Patients and methods}

\section{Patients}

The medical records of 85 patients with $\mathrm{HCC}$ who had received radical hepatectomy in our hospital from May 2005 to May 2010 were retrospectively analyzed. Baseline characteristics - age, sex, serum AFP level, operating time, blood loss during surgery, etiology, degree of cirrhosis, resection modalities, microvascular invasion (MVI), Child-Pugh stage, tumor size, number of tumors, and tumor differentiation - were collected and compared. The presence of MVI was determined by pathological findings from surgical specimens.

Among the patients, 42 underwent two courses of HAIC after radical hepatectomy (HAIC group) and 43 underwent radical hepatectomy without postoperative HAIC (control group). Entry criteria were 1) age 18-75 years, 2) Karnofsky performance score $\geq 70,3$ ) no allergy to the chemotherapy agent, 4) no previous anticancer therapy, 5) Child-Pugh class $\mathrm{A}-\mathrm{B}, 6)$ no portal vein thrombosis or systemic metastasis, 7) no refractory ascites or organ dysfunction, 8) no recurrence or remnant tumor detected within 1 month after radical hepatectomy, and 9) full follow-up data.

The protocol was approved by the ethics committee of the First People's Hospital, affiliated to Huzhou Normal College, and followed the principles of the Declaration of Helsinki and Good Clinical Practice guidelines. Signed informed consent was obtained from all patients.

\section{HAIC administration}

HAIC was administered within 3 weeks after surgery for two sessions, with an interval of 4 weeks. The procedure included oxaliplatin $\left(\mathrm{Oxa} ; 85 \mathrm{mg} / \mathrm{m}^{2}\right)$ as a 2-hour infusion, followed by fluorouracil (5FU; $1,000 \mathrm{mg} / \mathrm{m}^{2}$ ) as a 5 -hour infusion and mitomycin $\mathrm{C}\left(6 \mathrm{mg} / \mathrm{m}^{2}\right)$ as a $>1$-hour infusion. HAIC was administered via a catheter, with one tip inserted into the common hepatic artery or proper hepatic artery and the other end connected to the micropump. ${ }^{12}$ The catheter was removed after HAIC. Patients were carefully monitored, and symptomatic treatments were administered. Treatment would be terminated in cases of life-threatening side effects or upon the request of the patient.

\section{Assessment and follow-up}

All complications and toxicities assessed according to the Common Terminology Criteria for Adverse Events (version 4.0) were recorded. Patients were followed up every 4 weeks in the first year after surgery and every 3 months thereafter. The follow-up assessment included physical examination, biological testing, AFP level, chest radiography, abdominal ultrasonography and computed tomography, or magnetic resonance imaging. Recurrence was diagnosed by imaging and if necessary biopsy. Once recurrence or metastasis was confirmed, transcatheter arterial chemoembolization, radiofrequency ablation, or surgery was performed.

\section{Statistical analysis}

Data are expressed as means \pm SD. Statistical analysis was conducted using the two-sample $t$-test and adjusted $\chi^{2}$ test between the two groups. Fisher's exact test was also employed if individual cell size was fewer than five counts. Survival probabilities were calculated using the Kaplan-Meier method, and survival curves were analyzed using the log-rank test. $P<0.05$ was considered statistically significant.

\section{Results \\ Baseline characteristics}

Baseline characteristics are listed in Table 1. No significant differences in age, sex, serum AFP level, operating time, blood loss during surgery, etiology, degree of cirrhosis, resection modalities, MVI, Child-Pugh stage, tumor size, number of tumors, or tumor differentiation were indicated.

\section{Intrahepatic recurrence-free survival}

In the first 5 postoperative years, 23 of 42 patients in the HAIC group and 29 of 43 patients in the control group developed intrahepatic recurrence. The HAIC group obtained significantly higher intrahepatic recurrence-free survival probability (HR 0.5615 , 95\% CI 0.3234-0.9749 [log-rank test]; $P=0.0332$ ) (Figure 1$)$.

\section{Disease-free survival}

In the first 5 postoperative years, 29 patients in the HAIC group and 36 patients in the control group developed recurrence. The 5-year disease-free survival probability was significantly higher in the HAIC group than in the control group (HR 0.591, 95\% CI 0.3613-0.9666 [log-rank test]; $P=0.0298$ ) (Figure 2). 
Table I Baseline characteristics

\begin{tabular}{|c|c|c|c|}
\hline & $\begin{array}{l}\text { Control } \\
(n=43)\end{array}$ & $\begin{array}{l}\text { HAIC } \\
(n=42)\end{array}$ & $P$-value \\
\hline Age (years) & $58.4 \pm 5.7$ & $59.1 \pm 6.2$ & 0.5892 \\
\hline $\operatorname{AFP}(\mathrm{ng} / \mathrm{mL})$ & $547.5 \pm 49.2$ & $562.4 \pm 54.1$ & 0.1875 \\
\hline Tumor size $(\mathrm{cm})$ & $5.7 \pm 1.3$ & $6.2 \pm 1.5$ & 0.1041 \\
\hline Operating time (minutes) & $187.2 \pm 44.1$ & $178.4 \pm 47.6$ & 0.379 \\
\hline Blood loss in operation $(\mathrm{mL})$ & $478.4 \pm 87.6$ & $492.5 \pm 108.9$ & 0.512 \\
\hline Sex & & & 0.6789 \\
\hline Male & 30 & 31 & \\
\hline Female & 13 & 11 & \\
\hline Microvascular invasion & & & 0.4172 \\
\hline Positive & 25 & 28 & \\
\hline Negative & 18 & 14 & \\
\hline Etiology & & & 0.8844 \\
\hline HBV & 24 & 25 & \\
\hline $\mathrm{HCV}$ & 7 & 8 & \\
\hline Alcohol & 7 & 6 & \\
\hline Others & 5 & 3 & \\
\hline Cirrhosis & & & 0.9008 \\
\hline Absent & 10 & 9 & \\
\hline Mild & 22 & 20 & \\
\hline Severe & 11 & 13 & \\
\hline Resection modalities & & & 0.7132 \\
\hline Single segmentectomy & 14 & 12 & \\
\hline Multiple segmentectomies & 20 & 18 & \\
\hline Lobectomy & 9 & 12 & \\
\hline Tumor number & & & 0.4766 \\
\hline Single & 24 & 23 & \\
\hline Multiple & 18 & 20 & \\
\hline Tumor differentiation & & & 0.7531 \\
\hline Good & 10 & 12 & \\
\hline Moderate & 12 & 14 & \\
\hline Poor & 20 & 17 & \\
\hline Child-Pugh stage & & & 0.5951 \\
\hline A & 27 & 24 & \\
\hline$B$ & 16 & 18 & \\
\hline
\end{tabular}

Note: Data presented as number or mean \pm standard deviation.

Abbreviations: AFP, alpha fetoprotein; HAIC, hepatic artery-infusion chemotherapy; $\mathrm{HBV}$, hepatitis B virus; $\mathrm{HCV}$, hepatitis $\mathrm{C}$ virus.

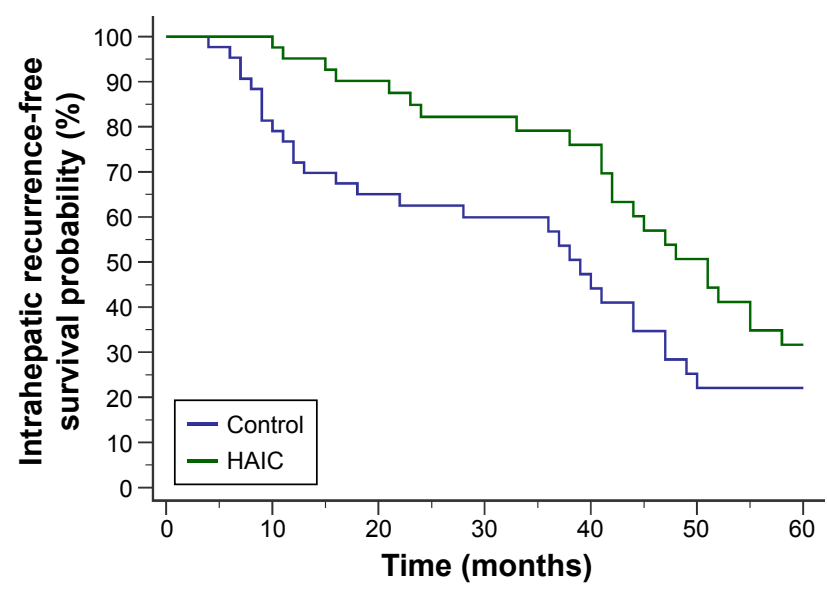

Figure I Intrahepatic recurrence-free survival curves after radical hepatectomy. Notes: In the first 5 postoperative years, 23 of 42 patients in the HAIC group and 29 of 43 in the control group developed intrahepatic recurrence. The HAIC group obtained significantly higher intrahepatic recurrence-free survival probability (HR 0.56I5, 95\% Cl 0.3234-0.9749; $P=0.0332$ ).

Abbreviation: HAIC, hepatic artery-infusion chemotherapy.

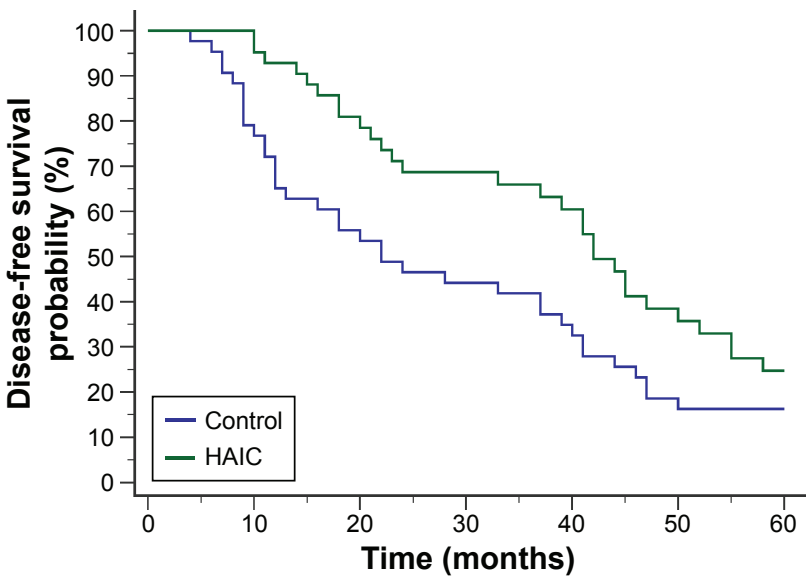

Figure 2 Disease-free survival curves after radical hepatectomy.

Notes: In the first 5 postoperative years, 29 patients in the HAIC group developed recurrence compared with 36 patients in the control group. The 5-year disease-free survival probability was significantly higher in the HAIC group than in the control group (HR 0.59I, 95\% Cl 0.36I3-0.9666, $P=0.0298$ ).

Abbreviation: HAIC, hepatic artery-infusion chemotherapy.

\section{Overall survival}

In the first 5 postoperative years, 27 patients in the HAIC group and 34 patients in the control group had died. The HAIC group obtained significantly higher overall survival probability than the control group (HR $0.5768,95 \%$ CI 0.3469-0.9589 [log-rank test]; $P=0.0278$ ) (Figure 3).

\section{Toxicity and complications}

No HAIC-induced deaths in the HAIC group were reported. Patients in the HAIC group mainly experienced paresthesia (14 of 42), neutropenia (15 of 42), thrombocytopenia (16 of 42), anemia (10 of 42), nausea or vomiting (30 of 42),

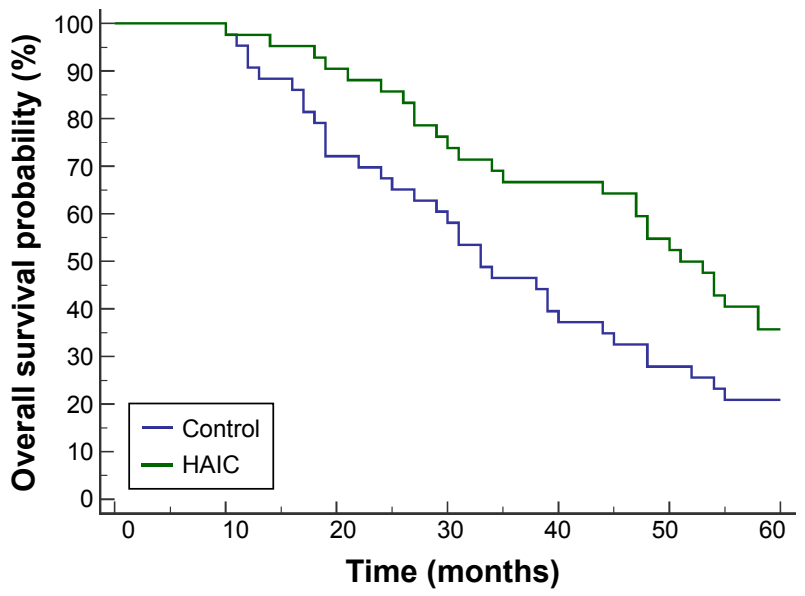

Figure 3 Overall survival curves after radical hepatectomy.

Notes: In the first 5 postoperative years, 27 patients in the HAIC group and 34 patients in the control group had died. The HAIC group obtained significantly higher overall survival probability than the control group (HR $0.5768,95 \% \mathrm{Cl} 0.3469-$ 0.9589; $P=0.0278$ ).

Abbreviation: HAIC, hepatic artery-infusion chemotherapy. 
diarrhea ( 8 of 42), and hepatic toxicity ( 25 of 42). All toxicities and complications were controlled, and no patients quit the treatment.

\section{Discussion}

HCC is a global public health problem, and causes millions of death annually worldwide. It is considered one of the most common cancers in China. ${ }^{13}$ Due to the difficulty of early diagnosis and the high frequency of metastasis and recurrence, the prognosis of $\mathrm{HCC}$ remains poor.

Liver tumors have been demonstrated to receive abundant blood supply from the hepatic artery. ${ }^{14-16}$ HAIC is a hepatically directed treatment that takes advantage of relatively selective hepatic arterial tumor vascularization. Several meta-analyses and clinical studies have concluded that postoperative HAIC can decrease recurrence after surgery and improve long-term survival. ${ }^{17-19}$ HAIC draws interest as a potential treatment option, because compared with intravenous infusion, extraction of chemotherapeutic drugs from the hepatic arterial circulation via the first-pass effect can produce higher local concentrations and fewer systemic side effects. Accordingly, we started the protocol of arterial infusion of Oxa $\left(85 \mathrm{mg} / \mathrm{m}^{2}\right.$ on day 1) followed by $5 \mathrm{FU}\left(1,000 \mathrm{mg} / \mathrm{m}^{2}\right.$ on day 1$)$ and mitomycin $\mathrm{C}\left(6 \mathrm{mg} / \mathrm{m}^{2}\right)$. The feasibility and safety of HAIC with Oxa, mitomycin $\mathrm{C}$, and 5FU for HCC treatment have been reported previously. ${ }^{20}$

In the current study, fewer patients in the HAIC group than in the control group developed intrahepatic recurrence during the first 5 years after surgery. The HAIC group obtained significantly higher 5 -year intrahepatic recurrence-free survival probability and lower risk of intrahepatic recurrence (HR $0.5615,95 \%$ CI $0.3234-0.9749 ; P=0.0332$ ). Consequently, the HAIC group also obtained significantly higher 5-year disease-free survival (HR 0.591, 95\% CI 0.3613-0.9666; $P=0.0298$ ) and overall survival probability compared with the control group (HR $0.5768,95 \%$ CI $0.3469-0.9589$ [logrank test]; $P=0.0278$ ). No HAIC-related deaths in the HAIC group were reported. No patients quit the treatment because of uncontrollable toxicities or complications.

In conclusion, HAIC is an effective and safe procedure for preventing postoperative intrahepatic recurrence and improving the long-term survival of patients with HCC after curative resection. The current protocol can be safely employed in a prospective randomized study.

\section{Acknowledgment}

This study was supported by the Foundation of Huzhou Bureau of Science and Technology (grant 2016YZB02).

\section{Author contributions}

MF, CWT, and JBS designed the study and wrote the manuscript. MF and CWT contributed equally to this study. WMF, YB, and YYZ conducted patient management and monitoring. YB and WMF collected and analyzed the data. All authors contributed toward data analysis, drafting, and revising the paper, and agree to be accountable for all aspects of the work.

\section{Disclosure}

The authors report no conflicts of interest in this work.

\section{References}

1. Jemal A, Bray F, Center MM, Ferlay J, Ward E, Forman D. Global cancer statistics. CA Cancer J Clin. 2011;61(2):69-90.

2. Miyaki D, Aikata H, Honda Y, et al. Hepatic arterial infusion chemotherapy for advanced hepatocellular carcinoma according to Child-Pugh classification. J Gastroenterol Hepatol. 2012;27(12):1850-1857.

3. Uka K, Aikata H, Takaki S, et al. Pretreatment predictor of response, time to progression, and survival to intraarterial 5-fluorouracil/interferon combination therapy in patients with advanced hepatocellular carcinoma. $J$ Gastroenterol. 2007;42(10):845-853.

4. Tang CW, Zhu M, Feng WM, Bao Y, Zheng YY. Chinese herbal medicine, jianpi ligan decoction, improves prognosis of unresectable hepatocellular carcinoma after transarterial chemoembolization: a retrospective study. Drug Des Devel Ther. 2016;10:2461-2466.

5. Imamura H, Matsuyama Y, Tanaka E, et al. Risk factors contributing to early and late phase intrahepatic recurrence of hepatocellular carcinoma after hepatectomy. J Hepatol. 2003;38(2):200-207.

6. Poon RT, Fan ST, Ng IO, Lo CM, Liu CL, Wong J. Different risk factors and prognosis for early and late intrahepatic recurrence after resection of hepatocellular carcinoma. Cancer. 2000;89(3):500-507.

7. Sitzmann JV, Abrams R. Improved survival for hepatocellular cancer with combination surgery and multimodality treatment. Ann Surg. 1993; 217(2):149-154.

8. Li Q, Wang J, Sun Y, et al. Postoperative transhepatic arterial chemoembolization and portal vein chemotherapy for patients with hepatocellular carcinoma: a randomized study with 131 cases. Dig Surg. 2006;23(4):235-240.

9. Cheng HY, Wang X, Chen D, Xu AM, Jia YC. The value and limitation of transcatheter arterial chemoembolization in preventing recurrence of resected hepatocellular carcinoma. World J Gastroenterol. 2005; 11(23):3644-3646.

10. Konishi M, Ryu M, Kinoshita T, Inoue K. Surgical treatment of hepatocellular carcinoma with direct removal of the tumor thrombus in the main portal vein. Hepatogastroenterology. 2001;48(41):1421-1424.

11. Tang C, Shen J, Feng W, et al. Combination therapy of radiofrequency ablation and transarterial chemoembolization for unresectable hepatocellular carcinoma: a retrospective study. Medicine (Baltimore). 2016; 95(20): e3754.

12. Kasugai H, Kojima J, Tatsuta M, et al. Treatment of hepatocellular carcinoma by transcatheter arterial embolization combined with intraarterial infusion of a mixture of cisplatin and ethiodized oil. Gastroenterology. 1989;97(4):965-971.

13. Bosch FX, Ribes J, Diaz M, Cleries R. Primary liver cancer: worldwide incidence and trends. Gastroenterology. 2004;127(5 Suppl 1): S5-S16.

14. Ackerman NB, Lien WM, Kondi ES, Silverman NA. The blood supply of experimental liver metastases - I: the distribution of hepatic artery and portal vein blood to "small" and "large" tumors. Surgery. 1969;66(6): 1067-1072.

15. Conway JG, Popp JA, Ji S, Thurman RG. Effect of size on portal circulation of hepatic nodules from carcinogen-treated rats. Cancer Res. 1983;43(7):3374-3378. 
16. Archer SG, Gray BN. Vascularization of small liver metastases. $B r J$ Surg. 1989;76(6):545-548.

17. Mathurin P, Raynard B, Dharancy S, et al. Meta-analysis: evaluation of adjuvant therapy after curative liver resection for hepatocellular carcinoma. Aliment Pharmacol Ther. 2003;17(10):1247-1261.

18. Tanaka K, Shimada H, Togo S, et al. Use of transcatheter arterial infusion of anticancer agents with Lipiodol to prevent recurrence of hepatocellular carcinoma after hepatic resection. Hepatogastroenterology. 1999;46(26):1083-1088.
19. Fukuda S, Okuda K, Imamura M, Imamura I, Eriguchi N, Aoyagi S. Surgical resection combined with chemotherapy for advanced hepatocellular carcinoma with tumor thrombus: report of 19 cases. Surgery. 2002;131(3):300-310.

20. Guthoff I, Lotspeich E, Fester C, et al. Hepatic artery infusion using oxaliplatin in combination with 5-fluorouracil, folinic acid and mitomycin C: oxaliplatin pharmacokinetics and feasibility. Anticancer Res. 2003;23(6D):5203-5208.

\section{Publish your work in this journal}

OncoTargets and Therapy is an international, peer-reviewed, open access journal focusing on the pathological basis of all cancers, potential targets for therapy and treatment protocols employed to improve the management of cancer patients. The journal also focuses on the impact of management programs and new therapeutic agents and protocols on

\section{Dovepress}

patient perspectives such as quality of life, adherence and satisfaction. The manuscript management system is completely online and includes a very quick and fair peer-review system, which is all easy to use. Visit http://www.dovepress.com/testimonials.php to read real quotes from published authors.

Submit your manuscript here: http://www.dovepress.com/oncotargets-and-therapy-journal 